\title{
Monte Carlo simulation of metal deposition on foreign substrates.
}

\author{
M.C. Giménez * A. J. Ramirez-Pastor \\ Laboratorio de Ciencias de Superficies y Medios Porosos, \\ Departamento de Física,
} Facultad de Ciencias Físico-Matemáticas y Naturales, Universidad Nacional de San Luis, CONICET, Chacabuco 917, 5700, San Luis, Argentina

\author{
E. P. M. Leiva \\ Unidad de Matemática y Física, Facultad de Ciencias Químicas, \\ Universidad Nacional de Córdoba, 5000 \\ Córdoba, Argentina
}

December 1, 2018

*Corrresponding author: M.C. Giménez, e-mail: cecigime@unsl.edu.ar 


\begin{abstract}
The deposition of a metal on a foreign substrate is studied by means of grand canonical Monte Carlo simulations and a lattice-gas model with pair potential interactions between nearest neighbors. The influence of temperature and surface defects on adsorption isotherms and differential heat of adsorption is considered. The general trends can be explained in terms of the relative interactions between adsorbate atoms and substrate atoms. The systems $A g / A u(100), A g / P t(100)$, $A u / A g(100)$ and $P t / A g(100)$ are analyzed as examples.
\end{abstract}

Keywords: metal deposition, lattice-gas model, Monte Carlo simulation.

\title{
1 Introduction
}

The study of the adsorption of particles on surfaces is important from the point of view of surface science and due to its potential applications in nanotechnology and catalysis.

From the electrochemical point of view, it is of great interest to study the electrodeposition of a metal onto a single crystalline surface of a foreign metal [1, 2, 3, 4]. When this occurs at potentials more positive than those predicted from the Nernst equation, the process is denominated underpotential deposition (UPD) [1, 3, 5, 6, 7]. This can be intuitively understood considering that, in general, UPD is observed when for the adsorbate it is more favorable to be deposited on the considered substrate than on a surface of its same nature. When the opposite occurs, the observation of another phenomenon 
called overpotential deposition (OPD) is expected, which implies that the adsorption of the adsorbate on the foreign substrate is less favorable than that predicted by the Nernst equation.

It is clear that a complete analysis of the UPD phenomenon is a quite difficult subject because of the complexity of the involved systems. For this reason, the understanding of simple models with increasing complexity might be a help and a guide to establish a general framework for the study of this kind of systems. Pioneering work using Monte Carlo simulations to study underpotential multisite adsorption has been undertaken by Van Der Eerden et al. [8].In this context, the present work tries to contribute to the comprehension of some essential characteristics of the metal deposition by means of a very simple model. For this purpose, we present simulations of metal adsorption on metal surfaces, employing a lattice-gas model with pair potential interactions characterized by a few parameters. Depending on the particular values assigned to the parameters, we can represent different metals involved. As illustrative examples we have simulated the following systems: $A g / A u(100)$ (that means: adsorption of silver atoms on gold (100) surfaces), $A g / P t(100), A u / A g(100)$ and $P t / A g(100)$.

In previous works [9, 10] we have used a more complicated model for the calculation of energies, employing the embedded atom method, which is more appropriate for metals in the sense that takes into account manybody interactions. The cost of introducing this precursor model is the lack of some experimental features presented by real systems. However, there are important physical motivations to pay this cost: a) this contribution allows to identify and characterize the most prominent features of this particular 
process; b) the study offers a general theoretical framework in which this kind of process can be studied and c) the conclusions obtained are interesting. The outline of the paper is as follows: In Section 2 we describe the lattice-gas model along with the simulation scheme. In Section 3 we present the results. Finally, the general conclusions are given in Section 4 .

\section{Model and simulation method}

\section{$2.1 \quad$ Lattice model}

Lattice models for computer simulations are of widespread use in studies of adsorption on surfaces, because they allow dealing with a large number of particles at a relatively low computational cost [11].

If the crystallographic misfit between the involved atoms is not important, it is a good approximation to assume that the adatoms adsorb on defined discrete sites on the surface, given by the positions of the substrate atoms. This is the case of the very well studied system $A g / A u(100)$ [12, 13, 14, 15].

Square lattices with periodical boundary conditions are used in the present work to represent the surface of the electrode. Each lattice point represents an adsorption site for an adsorbate or a substrate atom. The former may adsorb or desorb on each randomly selected site, while the latter may only move on the surface jumping from the selected site to a neighboring one. In this way, our model corresponds to an open system for one of its components, that is, the adsorbate. This has physical correspondence with the setup of the electrochemical experiment, where only the metal in equilibrium with 
its cations in solution may dissolve or be deposited in the potential range considered.

Concerning the adatoms of the same kind of the substrate, some considerations must be made regarding the existence of surface defects. These atoms may in principle move freely on the surface and minimize the free energy of the system by a number of mechanisms. For example, isolated substrate atoms may heal defects through their incorporation to a defective cluster, or small substrate islands may dissolve to join large ones, like shown in previous simulation work by Stimming and Schmickler [16]. Thus, different substrate structures can be imposed as initial conditions for each simulation. In the present case, we employ islands of substrate atoms on the surface obtained by means of simulated annealing techniques [9]. This was undertaken in order to emulate some of the defects that can be found on a real single crystal surfaces, like kink sites, steps, etc.

On purely energetic grounds, metal electrodeposition on clean metal surfaces with islands should take place according to the following sequence:

i)Decoration of the lower part of island edges. This is so because the binding energy is the lower(more negative), the higher the coordination of the site where a metal atom becomes adsorbed.

ii)Deposition on the rest of the flat surface around the islands.

iii)Some nucleation should occur at the top of the islands. This requires a more negative potential than that for island decoration(i) or flat surface filling(ii) because the border of the growing nuclei makes them less stable than a growing monolayer (nucleation overpotential)

iv)The border of the islands at the top should be finally decorated. These 
sites are particularly unstable due to their low coordination.

In the present work we are mainly interested in island decoration and the possible mixing between island atoms and depositing atoms following immediately after this decoration. This involves steps i) and ii) described above. Steps iii) and iv) could be of course simulated but this will not be considered in the present work.

\subsection{Energy calculations}

The energy $E$ of the system is related with the classical lattice-gas model with pair potential interactions between nearest neighbors:

$E=\sum_{\alpha}\left(\epsilon_{1} \delta_{c_{\alpha}, 1}+\epsilon_{2} \delta_{c_{\alpha}, 2}\right)+\sum_{\langle\alpha, \beta\rangle}\left[J_{11} \delta_{c_{\alpha}, 1} \delta_{c_{\beta}, 1}+J_{22} \delta_{c_{\alpha}, 2} \delta_{c_{\beta}, 2}+J_{12}\left(\delta_{c_{\alpha}, 1} \delta_{c_{\beta}, 2}+\delta_{c_{\alpha}, 2} \delta_{c_{\beta}, 1}\right)\right]$

where the symbol $\delta$ represents the Kronecker delta and $c_{\alpha}$ indicates the occupation number of the $\alpha$ site [corresponding to a pair of $(x, y)$ coordinates]. Unlike the classical lattice-gas model, the occupation number may assume three different values: 0,1 or 2 , if the corresponding site is empty, occupied by an atom of the same kind of the substrate or occupied by an adsorbate atom, respectively. The notation $\langle\alpha, \beta\rangle$ implies a sum taking account of all pairs of nearest neighbors. $\epsilon_{i}$ is the adsorption energy of a particle of type $i$ and $J_{i j}$ are the pairwise interactions between nearest neighbors.

The values of the parameters considered here for the different systems are summarized in table 1 . These values were obtained according to calculations 
employing the embedded atom model [17, 9, 10]. In a previous work [10], we have calculated the adsorption energies corresponding to the different environments of the adsorption site, taking into account first, second and third neighbors. Some of the possible configurations were ilustrated in Fig. 1 and corresponding energies were indicated in table 1 of reference [10]. The values employed in the present work for $\epsilon_{i}$ were based on the adsorption energies corresponding to configuration 1 (that is, adsorption on a site without neighbors). The values employed for the parameters $J_{i j}$ were based on the others configurations $(2,3$, etc., which correspond to different occupation of neighboring sites) assuming pair potential interactions between nearest neighbors and taking an average value.

\subsection{Grand Canonical Monte Carlo}

Square lattices of $M=L \times L$ adsorption sites with periodical boundary conditions are used here to represent the surface (in this case, $L=100$ ). In the case of adsorption isotherms on clean surfaces, the initial state consists of 2000 adsorbate atoms distributed at random (that makes a coverage degree of 0.2 ). For the case of adsorption in the presence of surface defects, the initial state is characterized by $N_{d}=1000$ atoms of the same nature as that of the substrate forming islands previously generated by simulated annealing [9, 10], and 2000 adsorbate atoms distributed at random. The islands of substrate atoms are useful to emulate surface defects like kink and step sites.

The simulation consists in the realization of a certain number of Monte Carlo Steps (MCS) in order to equilibrate the system and then another set 
of MCS evaluation, from time to time, the thermodynamic quantities of interest (like energy of the system or coverage degree) in order to obtain the average value. This is performed for fixed values of temperature and chemical potential (Grand Canonical ensemble).

Each MCS implies the realization of $M$ trials. Each trial consists on the random selection of one lattice site, with the realization of one of the three following processes:

a) If the site is empty (occupation 0), the creation of an adsorbate atom is attempted (yielding occupation 2).

b) If the site is occupied by an adsorbate atom (occupation 2), its desorption is attempted (occupation 0 ).

c) If the site is occupied by a substrate atom (occupation 1) a nearest neighbor site is selected at random. If this new site is empty, a move to this position is tried.

In the three cases, the change is accepted with probability:

$$
P=\min \left\{1, \exp \left[-\frac{\Delta E-\mu \Delta N_{a}}{k_{B} T}\right]\right\}
$$

where $\Delta E\left(\Delta N_{a}\right)$ represents the difference between the energies (number of adsorbed particles) of the final and initial states, $k_{B}$ is the Boltzmann constant, $T$ is the temperature and $\mu$ is the chemical potential. Note that the value of $\Delta N_{a}$ is +1 in case $\left.a\right),-1$ in case $b$ ) and 0 in case $c$ ). 
The algorithm described above has some differences with the algorithm employed in Refs. [9, 10]. In the present case, only the substrate atoms can move between adjacent sites, although this possibility is indirectly contemplated for adsorbate atoms in the case of desorption from one site and adsorption in a neighboring site. This difference is not essential, the main target was to save computational time.

\subsection{Some definitions}

\subsubsection{Coverage degree}

The coverage degree, $\theta(\mu)$, which is defined as the number of sites occupied by an adsorbate atom divided by the number of available sites (all adsorption sites that are not occupied by a substrate atom), is obtained as a simple average:

$$
\theta(\mu)=\frac{1}{M-N_{d}} \sum_{\alpha}\left\langle\delta_{c_{\alpha}, 2}\right\rangle=\frac{\left\langle N_{a}\right\rangle}{M-N_{d}}
$$

where $\left\langle N_{a}\right\rangle$ is the mean number of adsorbate atoms on the surface and $\langle\cdots\rangle$ denotes the time average over the Monte Carlo simulation runs.

In the case of adsorption in the presence of surface defects, that is, when some substrate islands are present, we also define some partial coverages, like the coverage degree of steps sites, $\theta_{s}$, and the coverage degree of kink sites, $\theta_{k}$ :

$$
\theta_{s}=\frac{\left\langle N_{s, o}\right\rangle}{\left\langle N_{s, t}\right\rangle}
$$




$$
\theta_{k}=\frac{\left\langle N_{k, o}\right\rangle}{\left\langle N_{k, t}\right\rangle}
$$

where $\left\langle N_{s, t}\right\rangle\left(\left\langle N_{k, t}\right\rangle\right)$ is the average number of step (kink) sites on the surface and $\left\langle N_{s, o}\right\rangle\left(\left\langle N_{k, o}\right\rangle\right)$ is the average number of step (kink) sites occupied by an adsorbate atom.

\subsubsection{Differential heat}

We calculate the differential heat of adsorption, defined as [18]:

$$
q_{d}=-\frac{\partial\langle E\rangle}{\partial\left\langle N_{a}\right\rangle}
$$

where $\langle E\rangle$ is the mean energy of the adlayer.

The physical interpretation of this quantity is the energy associated with removing a particle from the surface at each moment.

In the case of computer simulations, the most appropriate way of performing the calculation of this value is by means of averaged quantities. We employ the following formulation [11, 19]:

$$
q_{d}=-\frac{\left\langle E N_{a}\right\rangle-\langle E\rangle\left\langle N_{a}\right\rangle}{\left\langle N_{a}^{2}\right\rangle-\left\langle N_{a}\right\rangle^{2}}
$$

\subsubsection{Quantification of island disintegration}

As discussed below, for some systems the substrate islands are found to disintegrate upon adatom deposition. In order to quantify this phenomenon, we define some (normalized) quantities characterizing the disintegration of substrate islands present on the surface when the adsorbate particles adsorb, 
as follows:

- Fraction number of $1-0$ pairs:

$$
N P_{1-0}=\frac{\sum_{<\alpha, \beta>} \delta\left(1, c_{\beta}\right) \times \delta\left(0, c_{\alpha}\right)}{c N_{d}}
$$

where the sum yields the number of adjacent substrate atom-empty site pairs. $c$ is the lattice connectivity (in this case $c=4$ ).

- Fraction number of $1-2$ pairs:

$$
N P_{1-2}=\frac{\sum_{<\alpha, \beta>} \delta\left(1, c_{\beta}\right) \times \delta\left(2, c_{\alpha}\right)}{c N_{d}}
$$

where the sum yields the number of adjacent substrate-adsorbate pairs.

- Total number of $1-X$ pairs, with $X=0,2$, that we define from:

$$
N P_{T}=N P_{1-0}+N P_{1-2}
$$

which corresponds to the fraction of sites surrounding substrate atoms that are not occupied by particles of the same kind (type 1). The resulting quantity, further on called "fraction of island disintegration", is given by the number of substrate atoms/adsorbate atoms and substrate atoms/empty sites pairs.

The maximum value of $N P_{T}$ will be reached in the case where the islands are completely disintegrated, that is, when there is a minimum of neighboring substrate-substrate pairs. In principle, the upper value of $N P_{T}$ could be 
1, in the case where no substrate-substrate pairs occur at the maximum coverage by the adsorbate. However, if we only take into account statistical considerations, it is to be expected that the probability of finding a neighbor of the same species (1) should be given by the fraction $N_{d} / M$, which in the present simulation is fixed to the value equal to 0.1 . In other words, it is expected that in the limit of high temperatures where entropic effects prevail, and for large $\theta$, we should get that $N P_{T} \longrightarrow 0.9$.

For a given distribution of well equilibrated substrate islands, the minimum value of $N P_{T}$ will be given by the fraction of pairs involving atoms that belong to the edge of the island at beginning of the simulation. If the islands do not disintegrate at all, remaining intact, $N P_{T}$ should remain the same all over the simulation. On the other hand, if the islands disintegrate, $N P_{T}$ should reflect the increasing connectivity of the substrate atoms to sites of a diferent kind.

\section{Results and discussion}

\subsection{Adsorption on clean surfaces}

Adsorption isotherms were simulated for the systems $\mathrm{Ag} / \mathrm{Au}(100), \mathrm{Ag} / \mathrm{Pt}(100)$, $A u / A g(100)$ and $P t / A g(100)$. The corresponding curves are plotted in the upper part of Figs. 1 and $2(a)$ and $b)$ ), for different temperatures. It can be seen that at low temperatures the isotherms show an abrupt jump, typical of first order phase transitions. As the temperature increases, the isotherms become smoother, specially for the first two systems. 
The present model is analogous to the Ising model in the sense that lateral interaction between adsorbing particles is considered as a pair-potential and only between nearest neighbors. It is well known that in this case the critical temperature for $\theta=0.5$ can be estimated as [20]:

$$
T_{C}=\frac{J}{2 k_{B} \ln (\sqrt{2}-1)}
$$

where $J$ is the lateral interaction between adsorbing particles.

Taking into account the values of $J_{22}$ employed here (see table 1 ), the estimated critical temperatures are: $T_{C}=1843 \mathrm{~K}$ for the system $\mathrm{Ag} / \mathrm{Au}(100)$; $T_{C}=1382 \mathrm{~K}$ for $\mathrm{Ag} / \mathrm{Pt}(100) ; T_{C}=3028 \mathrm{~K}$ for $\mathrm{Au} / \mathrm{Ag}(100)$ and $T_{C}=3950 \mathrm{~K}$ for $\mathrm{Pt} / \mathrm{Ag}(100)$. This is in agreement with the appreciation of the isotherms in Figs. 1 and 2, where it can be seen that the critical temperature must be between $1000 K$ and $2000 K$ for the systems $A g / A u(100)$ and $A g / P t(100)$, close to $3000 \mathrm{~K}$ for $\mathrm{Au} / \mathrm{Ag}(100)$ and above $3000 \mathrm{~K}$ for $\mathrm{Pt} / \mathrm{Ag}(100)$.

The chemical potential at which the isotherms of figure 1 and 2 present the step, say $\mu_{S}$, provides a measure for the affinity of the adsorbate for the substrate. This quantity can be substracted from the corresponding binding energy of the adsorbate, and compared with the so-called underpotential shift $\Delta \phi_{\text {upd }}$, which was first defined by Kolb et al. [1] as the potential difference between the desorption peak of a monolayer of a metal $M$ adsorbed on a foreign substrate $S$ and the current peak corresponding to the dissolution of the bulk metal $M$. The magnitude of $\Delta \phi_{\mathbf{u p d}}$ is a measure of the affinity of the adsorbate for the substrate, as compared with the affinity of the adsorbate with itself, and can be written in terms of the chemical potential per parti- 
cle of the atom adsorbed on a foreign substrate, $\mu[(S) M]$, and the chemical potential of the same species in the bulk $\mu[(M) M]$, according to[21]:

$$
\Delta \phi_{\text {upd }}=\frac{1}{z e_{0}}(\mu[(M) M]-\mu[(S) M])
$$

In the present model, $\mu[(M) M]$ can be replaced by the cohesive energy of the adsorbate, $E_{2}^{c o h}$, and $\mu[(S) M]$ may be in turn substituted by the chemical potential at which the isotherm presents the step $\mu_{S}$, since this chemical potential correspond to the occurrence of the adsorbate phase.

$$
\left.\Delta \phi_{\mathbf{u p d}}^{T}(1 x 1)=\frac{1}{z e_{0}}\left(E_{2}^{c o h}-\mu_{S}\right]\right)
$$

where we have introduced the superscript $T$ to denote that this is a theoretical prediction for the $(1 x 1)$ adsorbate structure . On the other hand, the experimental estimation of the underpotential shift $\Delta \phi_{\mathbf{u p d}}^{\exp }$ can be made from:

$$
\Delta \phi_{\mathbf{u p d}}^{\exp }(1 x 1)=\phi(1 x 1)-\phi_{N e r n s t}
$$

where $\phi(1 x 1)$ denotes the potential at which the (1x1) adsorbate phase appears, and $\phi_{\text {Nernst }}$ is the reversible deposition potential for the electrolyte solution employed. The $\mathrm{Ag} / \mathrm{Au}(100)$ system has been considered by different groups [12, 14, 22]. Ikemiya et al reported $\phi_{\text {Nernst }}=-58 \mathrm{mV}$ and $\phi(1 x 1) \approx 180 \mathrm{mV}$ vs $\mathrm{Hg} / \mathrm{Hg}_{2} \mathrm{SO}_{4}$ for this sytem, thus yielding $\Delta \phi_{\mathbf{u p d}}^{\exp }(1 x 1) \approx$ $238 \mathrm{mV}$. In the case of the $\mathrm{Ag} / \mathrm{Pt}(100)$ system, Aberdam et al.23] reported that the peak for the deposition of the first monolayer of Ag was located 0.48 
$\mathrm{V}$ more positive than the Nernst reversible potential, so that we take this

value for $\Delta \phi_{\text {upd }}^{\exp }(1 x 1)$. We show in Table II these experimental values along with the present theoretical estimations and those of our previous work using the more accurate embedded atom method potentials. It can be appreciated that the pair potential approximation performs almost as well as the more sophisticated many body one. There is no evidence in the literature for underpotential deposition of $\mathrm{Au}$ on $\mathrm{Ag}(100)$ or for Pt underpotential deposition on $\mathrm{Ag}(100)$. A related system, Pt deposition on $\mathrm{Au}(100)$ has been measured by Waibel et al.[24], with the finding that Pt deposition takes place at overpotentials. Since the cohesive energy of $\mathrm{Au}$ is larger than that of $\mathrm{Ag}$, it is expected that if $\mathrm{Pt}$ upd is not found on $\mathrm{Au}(100)$, it will be even less probable on $\operatorname{Ag}(100)$.

The lower parts of Figs. 1 and 2 (parts $c$ ) and $d)$ ) show the differential adsorption heats. The qualitative form of the curves of $q_{d}$ vs. $\mu$ is similar to the adsorption isotherms ( $\theta$ vs. $\mu$ ). The observed values of $q_{d}$ are in agreement with the estimation of $q_{d} \approx-\epsilon_{2}$ at very low coverages (corresponding to the adsorption of particles in sites without neighbors) and $q_{d} \approx-\epsilon_{2}-4 \times J_{22}$ at high coverages (corresponding to the adsorption of particles in sites surrounded by four nearest neighbors of the same kind).

\subsection{Adsorption in the presence of substrate islands}

Figs. 3 and 5 show the adsorption isotherms for two of the four studied systems in the presence of surface defects (substrate atoms present in the monolayer forming islands). In the figures, the isotherms for the complete 
monolayer are plotted together with the isotherms of steps and kink sites for three different temperatures [parts $a), b)$ and $c$ )], as well as the differential adsorption heats for the three temperatures [part $d)]$.

It can be seen that for $A g / P t(100)$ (Fig. 3) and $A g / A u(100)$ (not shown), the kink sites are occupied first, then the step sites and finally the complete monolayer. For the other two systems, at $T=300 K$, there is no differentiation between the different kind of sites, but at greater temperatures a small difference arises (see parts $a)-c$ ) of Fig. 5 for the system $A u / A g(100)$, the system $\mathrm{Pt} / \mathrm{Ag}(100)$ is not shown, but the results are very similar).

Analyzing the behavior of the differential heat of adsorption [part $d)$ ], it can be seen that for the first two systems (see Fig. 3 for $A g / P t(100)$ ), at low temperatures, there are four differentiated stages, before the adsorption of the complete monolayer (last part). The first stage with $q_{d} \approx-\left(\epsilon_{2}+2 J_{12}\right)$ (filling of substrate kink sites); the second one with $q_{d} \approx-\left(\epsilon_{2}+J_{12}+J_{22}\right)$ (filling of step sites besides to another adsorbate particle); the third one with $q_{d} \approx-\left(\epsilon_{2}+2 J_{22}\right)$ (filling of an adsorbate kink site, that is, adsorption on terrace sites next to two adsorbate particles during monolayer growth) and the fourth one with $q_{d} \approx-\left(\epsilon_{2}+4 J_{22}\right)$ (monolayer completion). All these stages are illustrated in Fig. 4. These estimations are closest to the results of the simulations for the system $\mathrm{Ag} / \mathrm{Pt}(100)$.

For the other two systems (see Fig. 5 for $A u / A g(100)$ ) there are at first sight only two more or less well defined stages: before and after monolayer completion. In the first part, where only a few adsorbate particles come into the system, the differential heat can be written as $q_{d} \approx-\left[\epsilon_{2}+4\left(J_{12}-J_{11}\right)+\right.$ $2 J_{11}$ ]. This value corresponds to the replacement of a substrate particle by 
an adsorbate particle in the middle of an island and the positioning of the former at the edge of the island. The interpretation of the value of $q_{d}$ is not straightforward for monolayer completion.

Fig. 6 shows the final state of a portion of the simulation cell at three different chemical potentials for the system $A g / A u(100)$ in the presence of defects at two different temperatures. At $T=300 K$, the islands remain almost unchanged upon adsorbate deposition and the sites are filled following the order: 1) kinks; 2) steps; 3) terraces. At $T=1000 K$, the general tendency remains but the islands show a certain disintegration. The same behavior is found for the system $\mathrm{Ag} / \mathrm{Pt}(100)$ (not shown).

On the other hand, in the case of the system $A u / A g(100)$ at $T=300 K$ (upper part of Fig. 7), the islands do not change their shape significantly but some adsorbate atoms penetrate inside them. At $T=1000 K$ (lower part of Fig. 7) the islands disintegrate completely. The same general behavior is found for the system $\mathrm{Pt} / \mathrm{Ag}(100)$ (not shown).

Figs. 8 and 9 show the adsorption isotherms and the fraction of island disintegration (characterized by the value $N P_{T}$, defined in equation 10) in the presence of substrate islands at three different temperatures for two of the considered systems.

In the case of the system $A g / A u(100)$ (Fig. 8), it can be verified that, at low temperatures, the islands remain unchanged, since the value of $N P_{T}$ is constant along the whole isotherm. As expected, the value of $N P_{1-0}$ decreases and the value of $N P_{1-2}$ increases as the adsorbate atoms cover the edges of the islands. At higher temperatures, the value of $N P_{T}$ increases when the adsorbate atoms enter. That means that both atom classes mix with 
each other, that is, the substrate islands disintegrate. At an intermediate temperature $(T=1000 K)$ the value of $N P_{T}$ presents a maximum close to the critical chemical potential due to the greater mobility of the atoms in that situation.

For the system $\mathrm{Ag} / \mathrm{Pt}(100)$ (not shown) the general trend is very similar to the previous case.

The adsorption isotherms for the systems $A u / A g(100)$ (not shown) and Pt/Ag(100) at different temperatures (Fig. 9a) show that, as the temperature increases, the jump in coverage moves towards more negative chemical potentials. Considering the fraction of island disintegration, an important difference with respect to the other systems consists in that even at low temperatures the islands start to disintegrate when the adatoms are deposited (Fig. 9b). This is evident in the increase of the quantity $N P_{T}$. Another important detail to be emphasized is that for high temperatures island disintegration is considerable, even for low adsorbate coverages (Fig. 9d). As anticipated in section 2.4.3, it can be observed in Figs. $8 d$ and $9 d$ that for all systems $N P_{T} \longrightarrow 0.9$ in the limit of high temperatures and adsorbate coverage degrees.

The general picture that we get from the present results is that as long as the susbtrate has a binding energy that is considerably larger than that of the adsorbate, the islands of the former remain relatively unaltered upon adsorbate formation. As the binding energy of the substrate approaches that of the adsorbate or becomes smaller, the islands become unstable and disintegrate relatively easily. The study of $\mathrm{Pd}\left(E_{c o h}=-3.91 \mathrm{eV}\right)$ deposition on $\mathrm{Au}(100)\left(E_{c o h}=-3.93 \mathrm{eV}\right)$ shows in fact the formation of a surface alloy in 
the underpotential region[25]. The study of $\mathrm{Pt}\left(E_{c o h}=-5.77 \mathrm{eV}\right)$ deposition on $\mathrm{Au}(100)$ shows some remarkable features. Although surface alloying was not reported for this system[24], the Pt deposit exhibit irregular shapes and recent molecular dynamic simulations point towards the existence of a surface alloy[26].

\section{Conclusions}

In the present paper we revisit sytems previously simulated in Refs. [9, 10] with a different model for the metal-metal interactions. Instead of the computationally demanding many-body potentials employed there, we use here attractive pairwise aditive potential interactions between nearest neighbors with only a minimal set of parameters. While the main results remain qualitatively similar, the present modelling is considerably simple and workable in the framework of the pairwise additive potential lattice model. It also saves considerable computational time. Furthermore, we have considered here the influence of the temperature and some new quantities, like differential heat and fraction of island disintegration, that helped us in the understanding of the simulations.

The present formulation shows that the main characteristics to be taken into account is the strenght of the interactions between adsorbate atoms as compared with the interaction between substrate atoms. In this sense, we can divide the four systems studied as examples into two groups. The first group is integrated by the systems $A g / A u(100)$ and $A g / P t(100)$. In these cases the interactions between adsorbate atoms is weaker than the interaction 
between substrate atoms (i.e., $\left|J_{22}\right|<\left|J_{11}\right|$ ). The second group is integrated by the systems $A u / A g(100)$ and $P t / A g(100)$, where the interactions between adsorbate atoms is stronger than the interaction between substrate atoms (i.e., $\left.\left|J_{22}\right|>\left|J_{11}\right|\right)$.

For defect-free surfaces, the adsorption isotherms show an abrupt jump at low temperatures and become smoother at high temperatures. This is indicative of the existence of a first order phase transition and a critical temperature related with the interaction between adsorbate atoms.

The adsorption in the presence of islands of the same nature as that of the substrate on the surface was also studied.

For systems in which the interaction between adsorbate particles is weaker than the interaction between substrate particles $(A g / A u(100)$ and $A g / P t(100))$, the substrate islands remain relatively unchanged at low temperatures and show a certain degree of mobility at high temperatures. For systems in which the interaction between adsorbate particles is stronger than the interaction between substrate particles $(A u / A g(100)$ and $P t / A g(100))$, the adsorbate atoms penetrate into the islands at low temperatures and the islands are completely disintegrated at high temperatures.

The simplicity of the present formulation will allow the analysis of problem at hand in terms of a few parameters than can be systematically varied. In other words, instead of system-oriented simulations, studies with these control parameters can be performed, with the consequent gain of generality. 


\section{Acknowledgements}

Financial support from CONICET, Agencia Córdoba Ciencia, Secyt U.N.C., Program BID 1201/OC-AR PICT No 06-12485 is gratefully acknowledged. M.C. Giménez thanks CONICET for a postdoctoral fellowship.

\section{References}

[1] D.M. Kolb, M. Przasnyski, H. Gerischer, J. Electroanal. Chem. 54 (1974) 25.

[2] S. Trasatti, Zeits. für Phys. Chem. NF 98 (1975) 75.

[3] E. Budevski, G. Staikov, W. J. Lorenz, "Electrochemical Phase Formation and Growth", VCH Weinheim, 1996.

[4] Budevski, E., Staikov, G., Lorenz, W.J., Electrochimica Acta 45 (15-16) (2000) 2559-2574.

[5] E. P. M. Leiva, in "Current Topics in Electrochemistry", Vol. 2, pag. 269, Council of Scientific Information, Trivandrum, India,(1993).

[6] E. P. M. Leiva, Electrochim. Acta 41 (1996) 2185.

[7] Plieth, W., Lorenz, W.J., Staikov, G., Journal of Solid State Electrochemistry 8 (12) (2004) 941-946.

[8] J.P. Van Der Eerden, G. Staikov, D. Kashchiev, W.J. Lorenz and E. Budevski, Surface Science 82(1979)364-382. 
[9] M. C. Giménez, M. G. Del Pópolo, E. P. M. Leiva, Electrochimica Acta 45 (1999) 699-712.

[10] M. C. Giménez, E. P. M. Leiva, Langmuir 19 (2003) 10538-10549.

[11] D. Nicholson and N. G. Parsonage, "Computer Simulation and Statistical Mechanics of Adsorption", Academic Press, (1982).

[12] S. G. García, D. Salinas, C. Mayer, E. Schmidt, G. Staikov, W. J. Lorenz, Electrochim. Acta 43 (1998) 3007.

[13] M.J. Esplandiu, M.A. Schneeweiss, D.M. Kolb, Phys. Chem. Chem. Phys. 1 (1999) 4847.

[14] S.G. García, D. Salinas, C. Mayer, J.R. Vilche, H.-J. Pauling, S. Vinzelberg, G. Staikov, W.J. Lorenz, Surface Science 316 (1994) 143-156.

[15] N. Ikemiya, K. Yamada, S. Hara, Surface Science 348 (1996) 253-260.

[16] W. Schmickler, U. Stimming, J. Electroanal. Chem. 366 (1994) 203.

[17] S. M. Foiles, M. I. Baskes, M. S. Daw, Phys. Rev. B 33 (1986) 7983.

[18] David M. Razmus and Carol K. Hall, AIChE Journal, Vol. 73, No. 5 (1991) 769-779.

[19] F. Bulnes, A. J. Ramirez-Pastor, Physica A 295 (2001) 71.

[20] Terrell L. Hill, "An Introduction to Statistical Thermodynamics", Dover publications, Inc., 1986. 
[21] D.M. Kolb in Advances in Electrochemistry and Electrochemical Engineering (Edited by H. Gerischer and C. W. Tobias), Vol. 11, p. 125, Wiley, New York (1978).

[22] N. Ikemiya, K. Yamada, S. Hara, Surface Science 348(1996)253.

[23] D. Aberdam, C. Salem, R. Durand, R. Faure, Surface Science 239(1990)71.

[24] H.-F. Waibel, M. Kleinert, L.A. Kibler, D.M. Kolb, Electrochim. Acta $47(2002) 1461$.

[25] L.A. Kibler, M. Kleinert,.M. Kolb, Surface Science 461(2000)155.

[26] W. Schmickler, K. Pötting, M. Mariscal, Chem. Phys. 320(2006)149. 


\section{$6 \quad$ Figure Captions}

Figure 01 a) Adsorption isotherms at different temperatures for the system $\mathrm{Ag} / \mathrm{Au}(100)$. b) Adsorption isotherms at different temperatures for the system $\mathrm{Ag} / \mathrm{Pt}(100)$. c) Differential heats for the adsorption isotherms of the system $A g / A u(100)$. d) Differential heats for the adsorption isotherms of the system $\mathrm{Ag} / \mathrm{Pt}(100)$.

Figure 02 a) Adsorption isotherms at different temperatures for the system $A u / A g(100)$. b) Adsorption isotherms at different temperatures for the system $\mathrm{Pt} / \mathrm{Ag}(100)$. c) Differential heats for the adsorption isotherms of the system $A u / A g(100)$. d) Differential heats for the adsorption isotherms of the system $\mathrm{Pt} / \mathrm{Ag}(100)$.

Figure $03 a)-c$ ) Adsorption isotherms in the presence of surface defects for the complete monolayer, the step sites and the kink sites at three different temperatures for the system $A g / P t(100) . d)$ : differential heat of adsorption at the three temperatures for the same system.

Figure 04 Schematic representation of the top view of four environment types close to the adatom adsorption site. $a$ ): substrate kink site. $b$ ): substrate step site, next to another adsorbate particle. c): adsorbate kink site. d): hollow site (upon monolayer completion). Filled circles denote substrate atoms. Empty circles represent adsorbate atoms. The empty dashed circle denotes the adsorption site under consideration. The underlying substrate atoms are not shown.

Figure $05 a)-c$ )Adsorption isotherms in the presence of surface defects for the complete monolayer, the step sites and the kink sites at three different 
temperatures for the system $A u / A g(100)$. d): differential heat of adsorption at the three temperatures for the same system.

Figure 06 Snapshots showing the final state of the surface at different chemical potentials for the system $A g / A u(100)$ in the presence of surface defects at $T=300 K$ and $T=1000 K$. Filled circles represent gold atoms while non-filled ones represent silver atoms.

Figure 07 Snapshots showing the final state of the surface at different chemical potentials for the system $A u / A g(100)$ in the presence of surface defects at $T=300 K$ and $T=1000 K$. Filled circles represent silver atoms while non-filled ones represent gold atoms.

Figure 08 . a)Adsorption isotherms in the presence of surface defects for the complete monolayer at three different temperatures for the system $A g / A u(100) . b)-d)$ Fraction number of pairs $\left(N P_{1-0}, N P_{1-2}\right.$ and $\left.N P_{T}\right)$ at the same temperatures.

Figure 09 a): Adsorption isotherms in the presence of surface defects for the complete monolayer at three different temperatures for the system $P t / A g(100) . b)-d)$ Fraction number of pairs $\left(N P_{1-0}, N P_{1-2}\right.$ and $\left.N P_{T}\right)$ at the same temperatures. 


\section{Tables}

\begin{tabular}{|c|c|c|c|c|c|}
\hline System & $\epsilon_{1}$ & $\epsilon_{2}$ & $J_{11}$ & $J_{22}$ & $J_{12}$ \\
\hline \hline$A g / A u(100)$ & -3.05 & -2.58 & -0.54 & -0.28 & -0.42 \\
\hline$A g / P t(100)$ & -4.34 & -3.13 & -0.83 & -0.21 & -0.56 \\
\hline$A u / A g(100)$ & -2.39 & -3.11 & -0.25 & -0.46 & -0.36 \\
\hline$P t / A g(100)$ & -2.30 & -4.22 & -0.17 & -0.60 & -0.41 \\
\hline
\end{tabular}

Table 1: Parameters representing the adsorption energies and the interaction energies (in $\mathrm{eV}$ units) employed here for the considered systems.

$\begin{array}{llll}\text { System } & \Delta \phi_{\mathbf{u p d}}^{T}(1 x 1) / V & \Delta \phi_{\mathbf{u p d}}^{T}(1 x 1), E A M / V & \Delta \phi_{\mathbf{u p d}}^{\exp }(1 x 1) / V \\ A g / A u(100) & 0.23 & 0.17 & 0.24^{a} \\ A g / P t(100) & 0.67 & 0.55 & 0.48^{b} \\ A u / A g(100) & 0.04 & -0.08 & <0 ?^{c} \\ P t / A g(100) & -0.44 & -0.53 & <0 ?^{c}\end{array}$

Table 2: Calculated and experimental underpotential shifts for the systems considered in the present work. $\Delta \phi_{\mathbf{u p d}}^{T}(1 x 1)$ denote results of this work, $\Delta \phi_{\text {upd }}^{T}(1 x 1) / E A M$ are results from previous simulations using the many-body potential from the embedded atom method[17], $\Delta \phi_{\mathbf{u p d}}^{\exp }(1 x 1)$ are experimental estimations taken from the literature. $\Delta \phi_{\mathbf{u p d}}^{T}(1 x 1)$ values were calculated using equation (13), with $E_{2}^{c o h}=-2.85,-3.93$ and $-5.77 \mathrm{eV}$ for $\mathrm{Ag}$, $\mathrm{Au}$ and $\mathrm{Pt}$ respectively.

a)taken from reference [22, b)Taken from reference [23] c)no upd has been reported in the literature for these systems so far. 

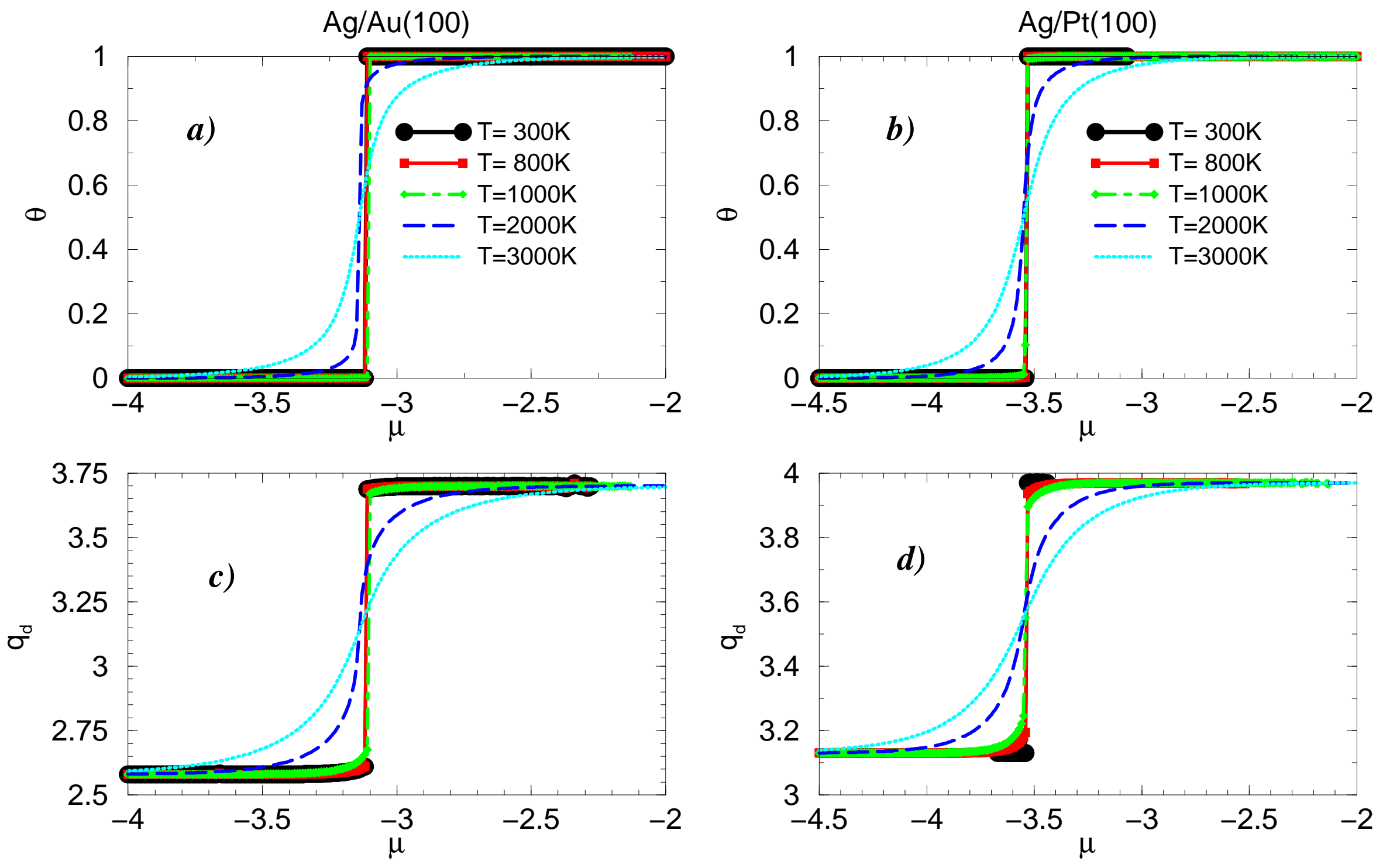

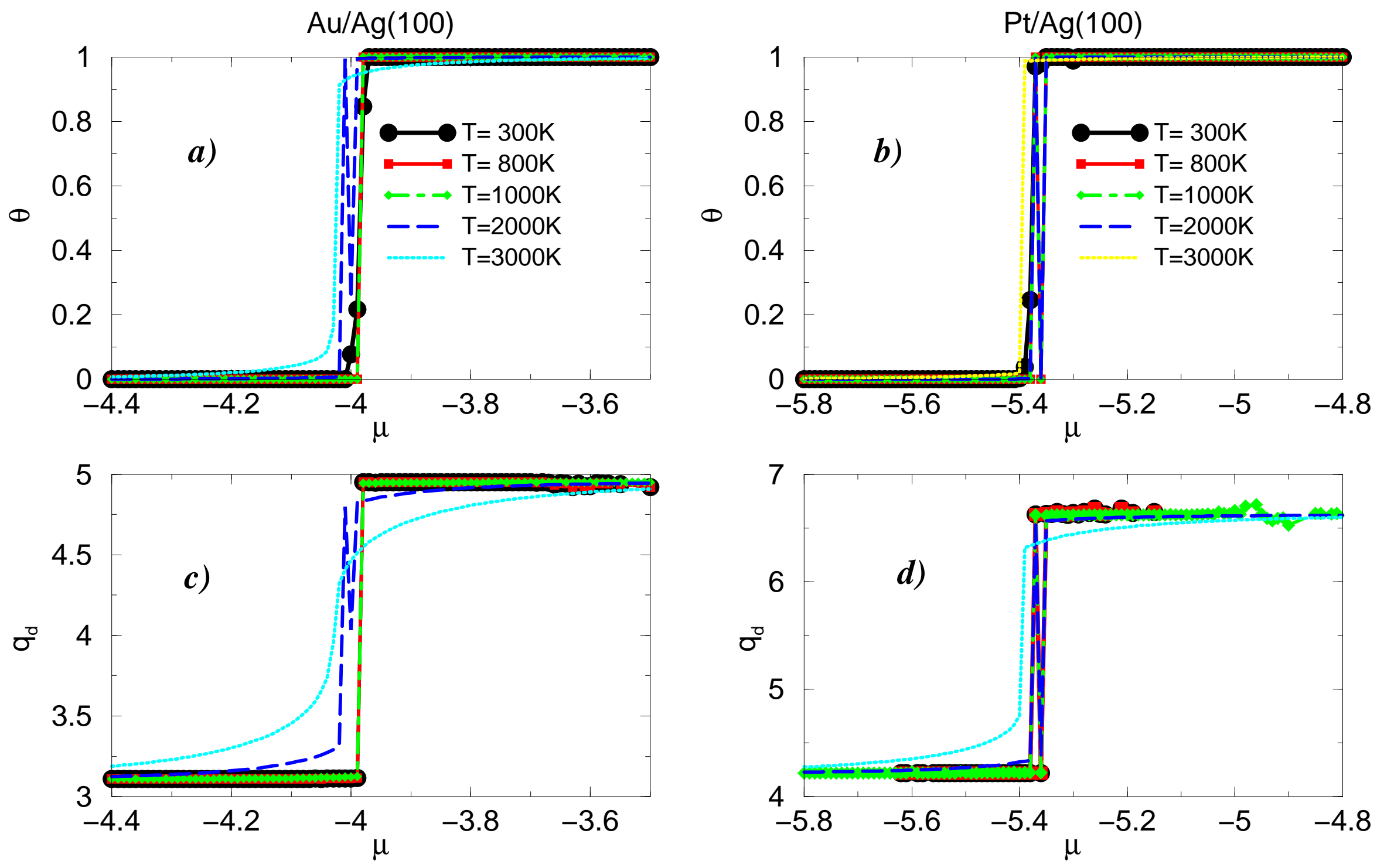


\section{$\mathrm{Ag} / \mathrm{Pt}(100)$}
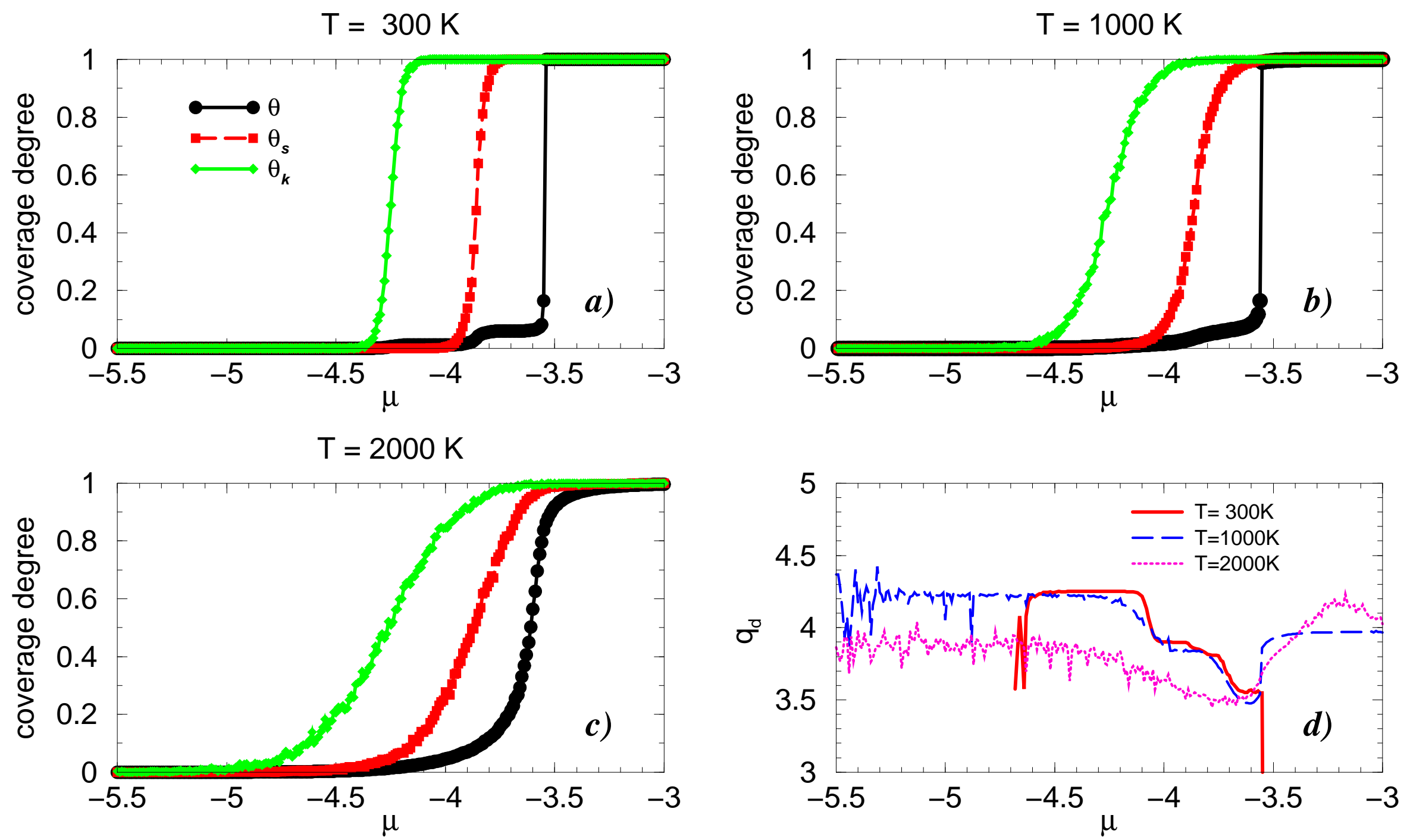

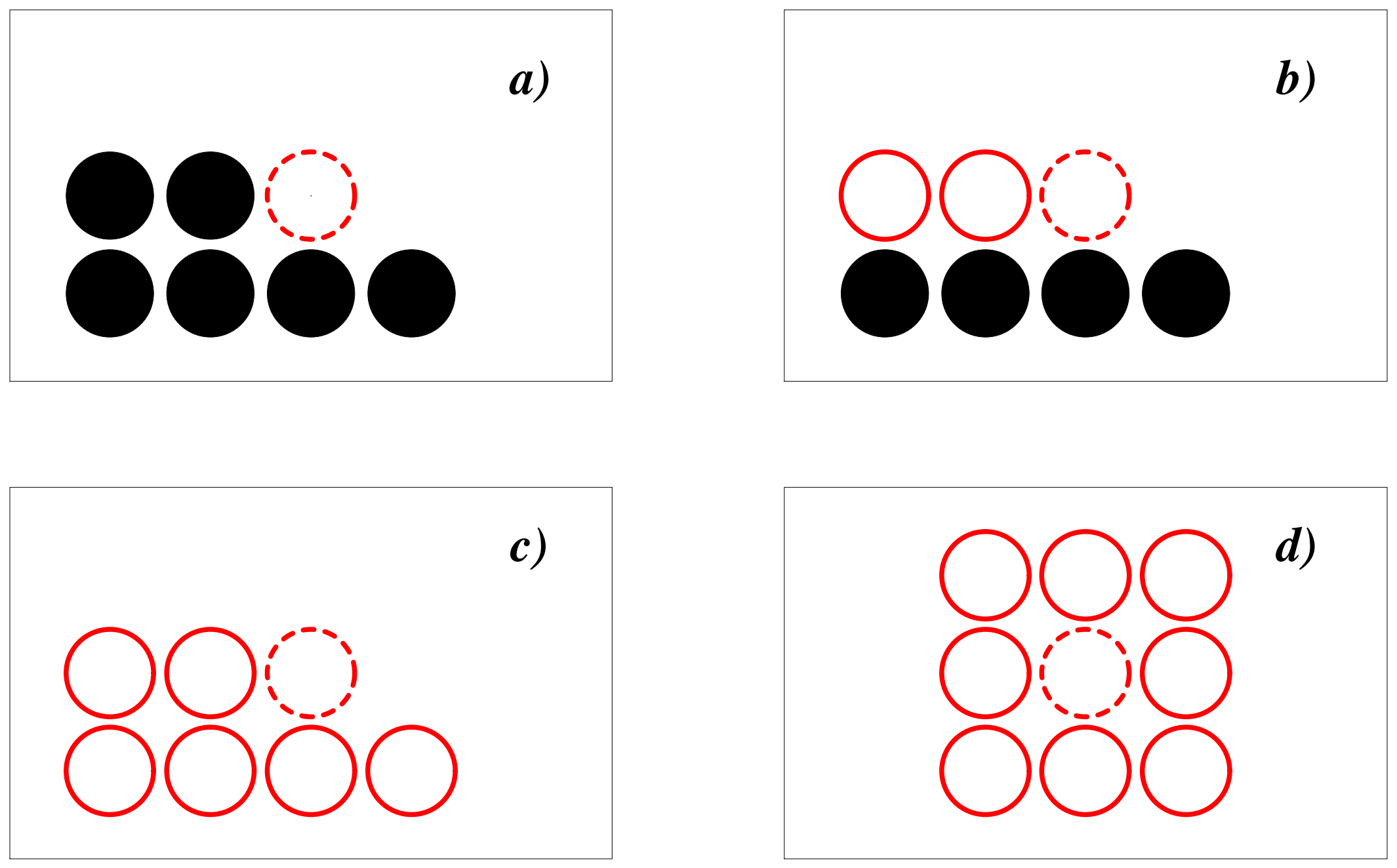
$\mathrm{Au} / \mathrm{Ag}(100)$
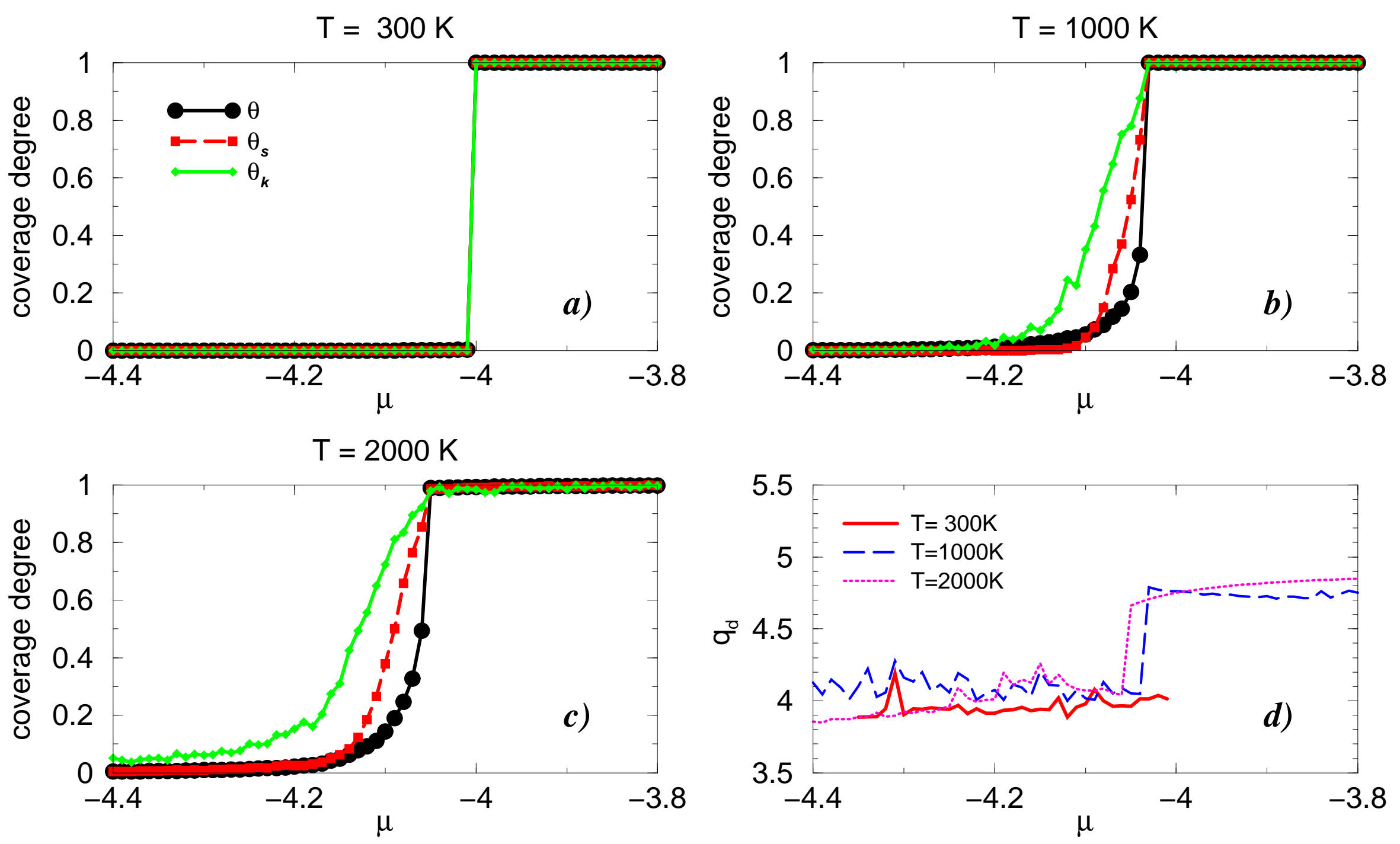

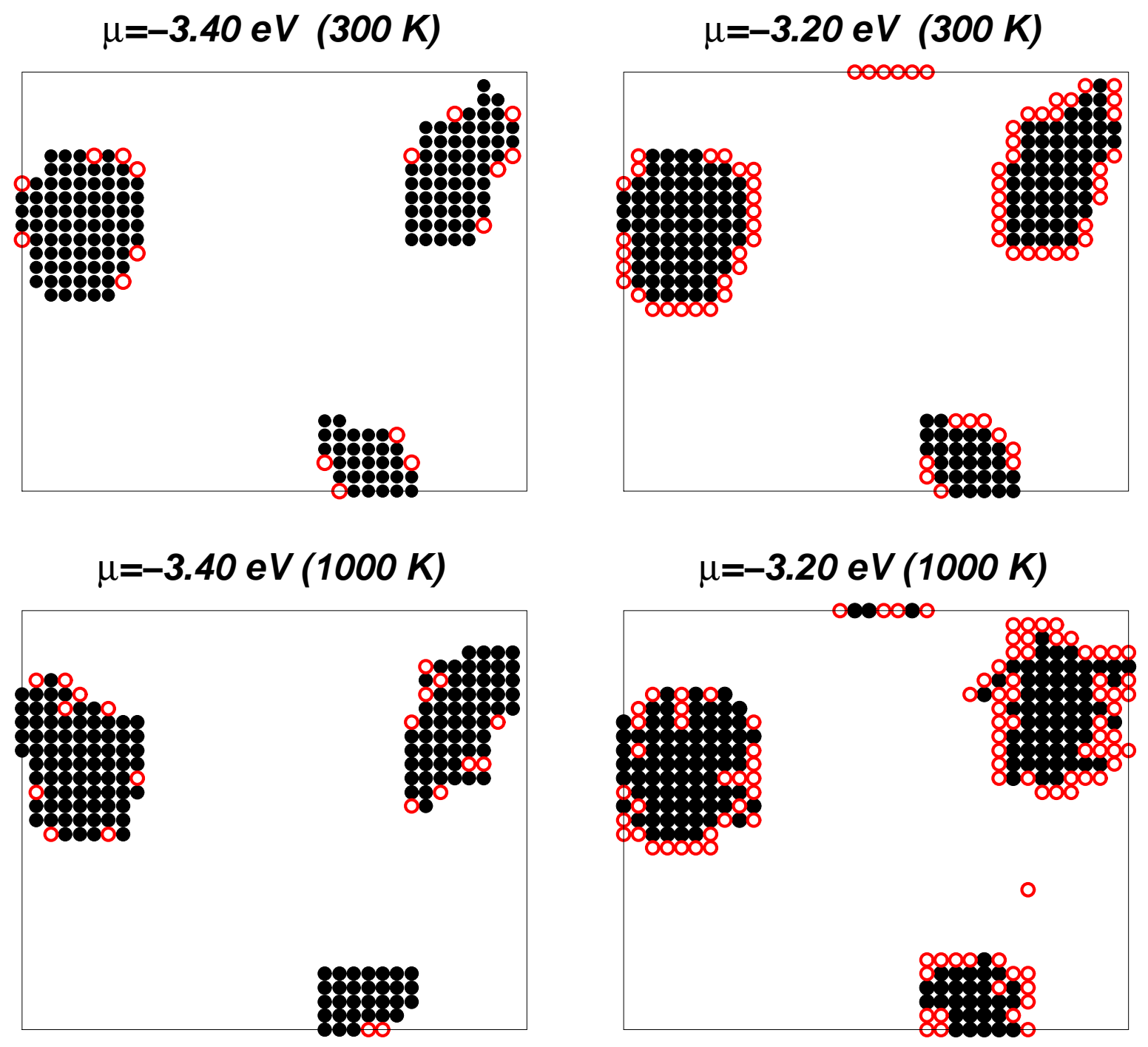
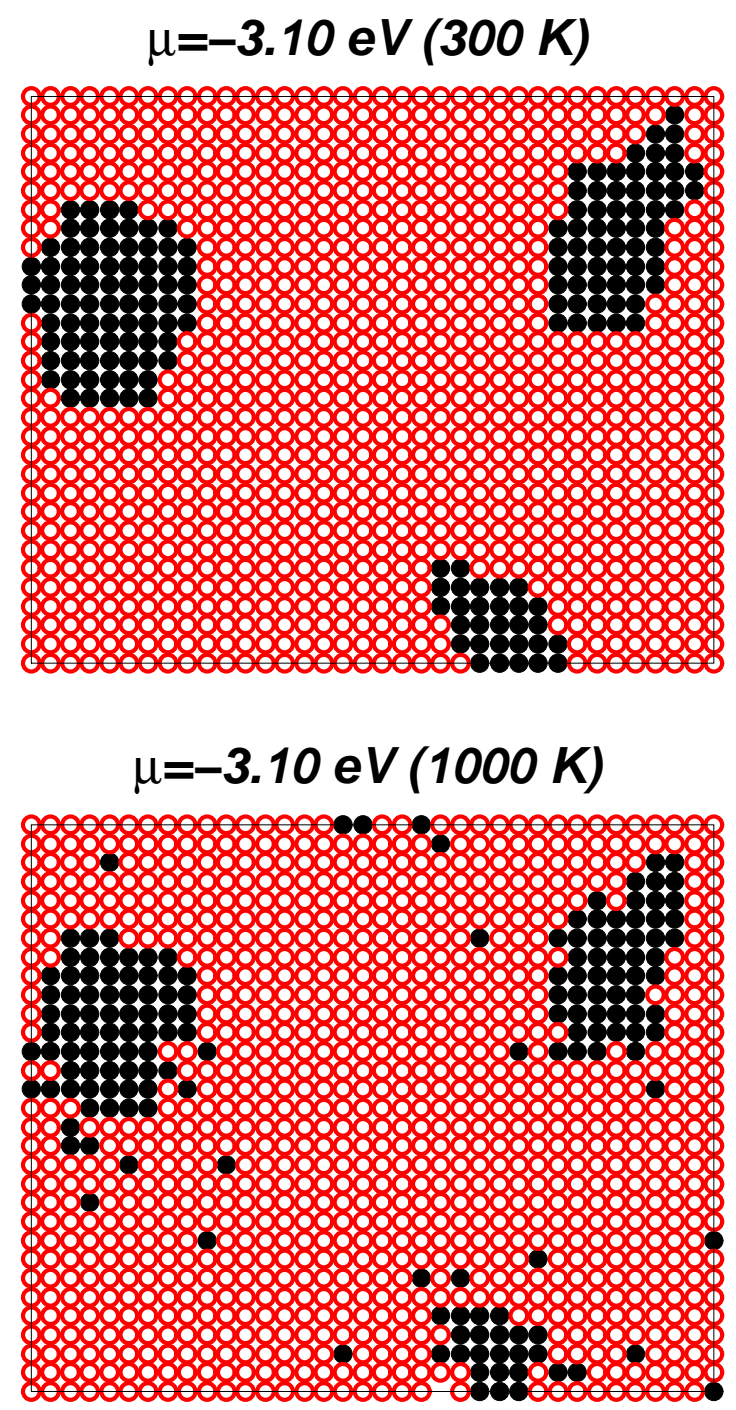

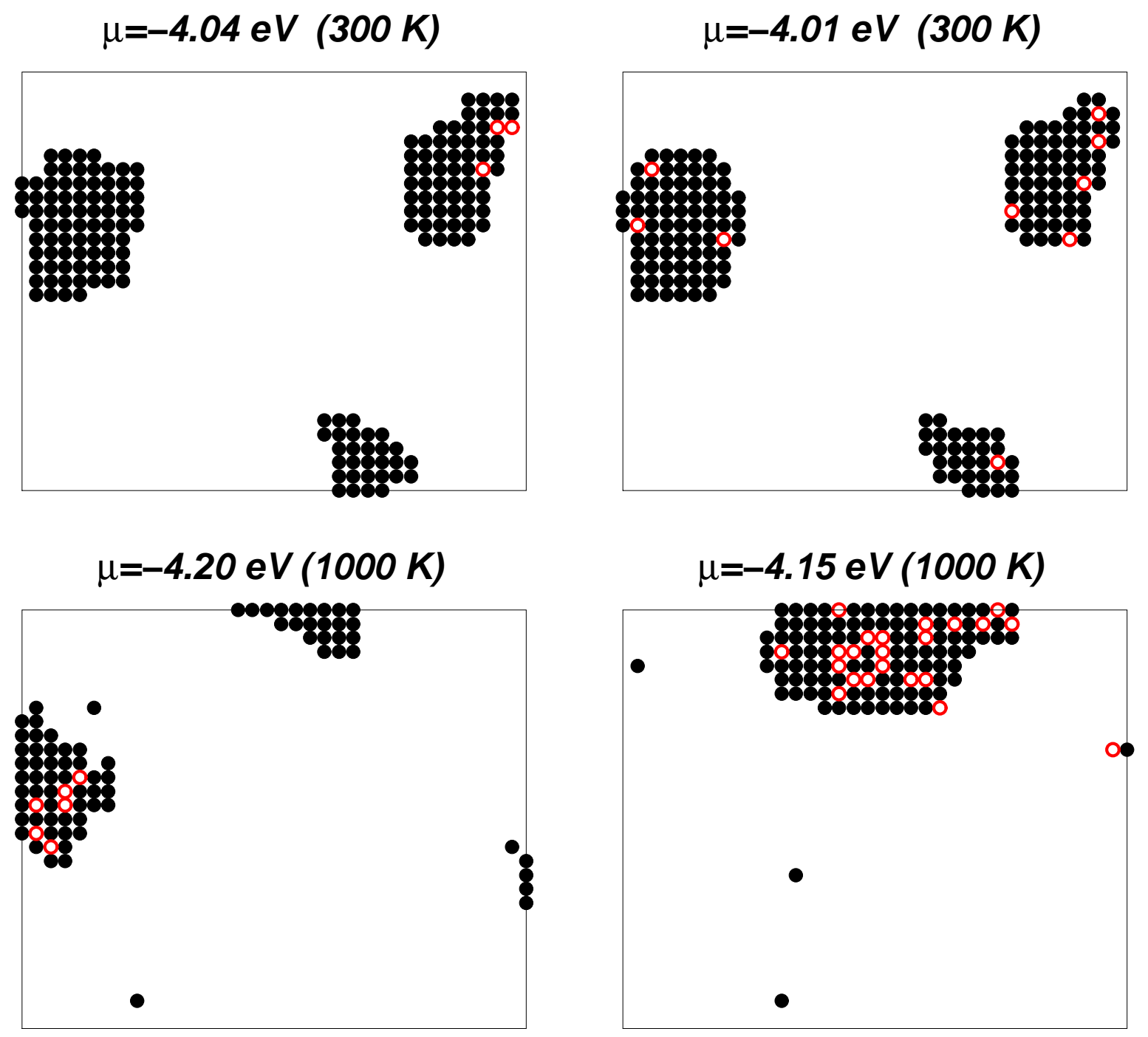
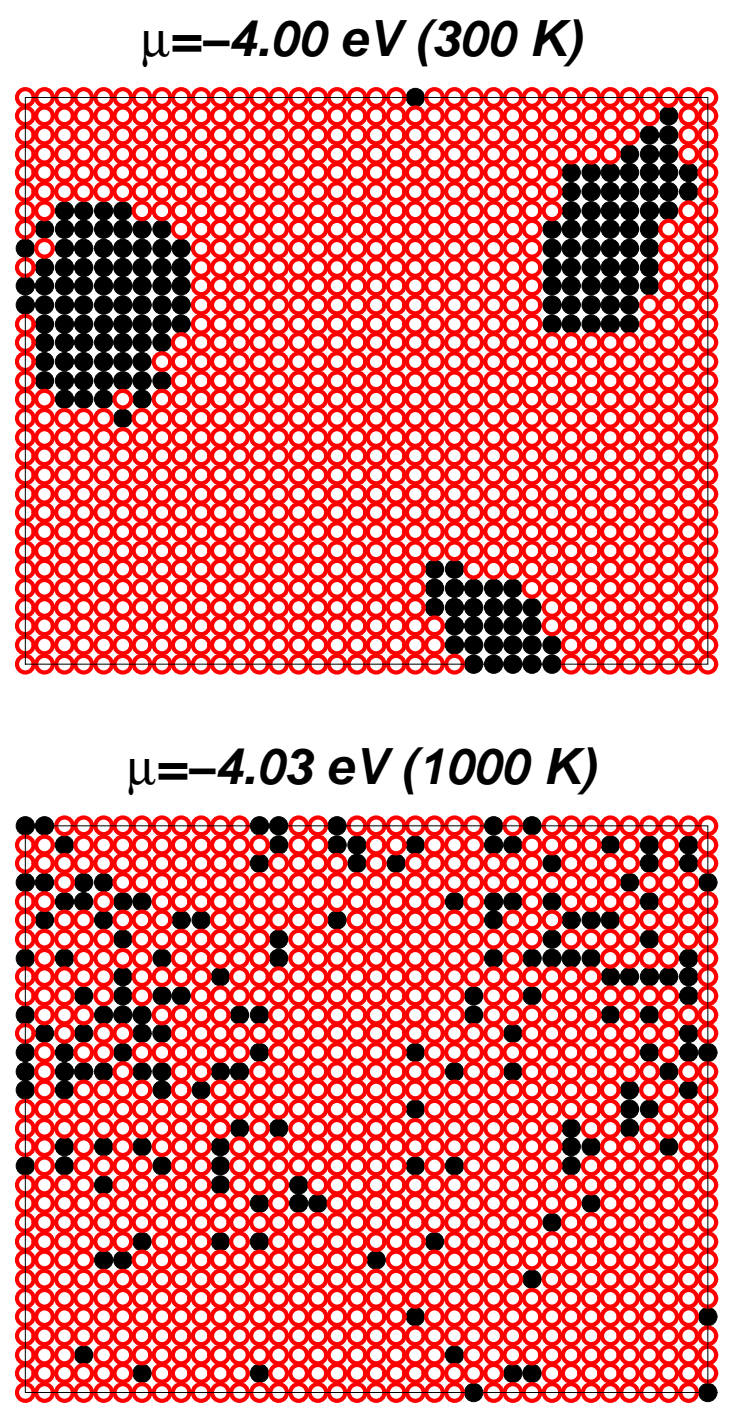

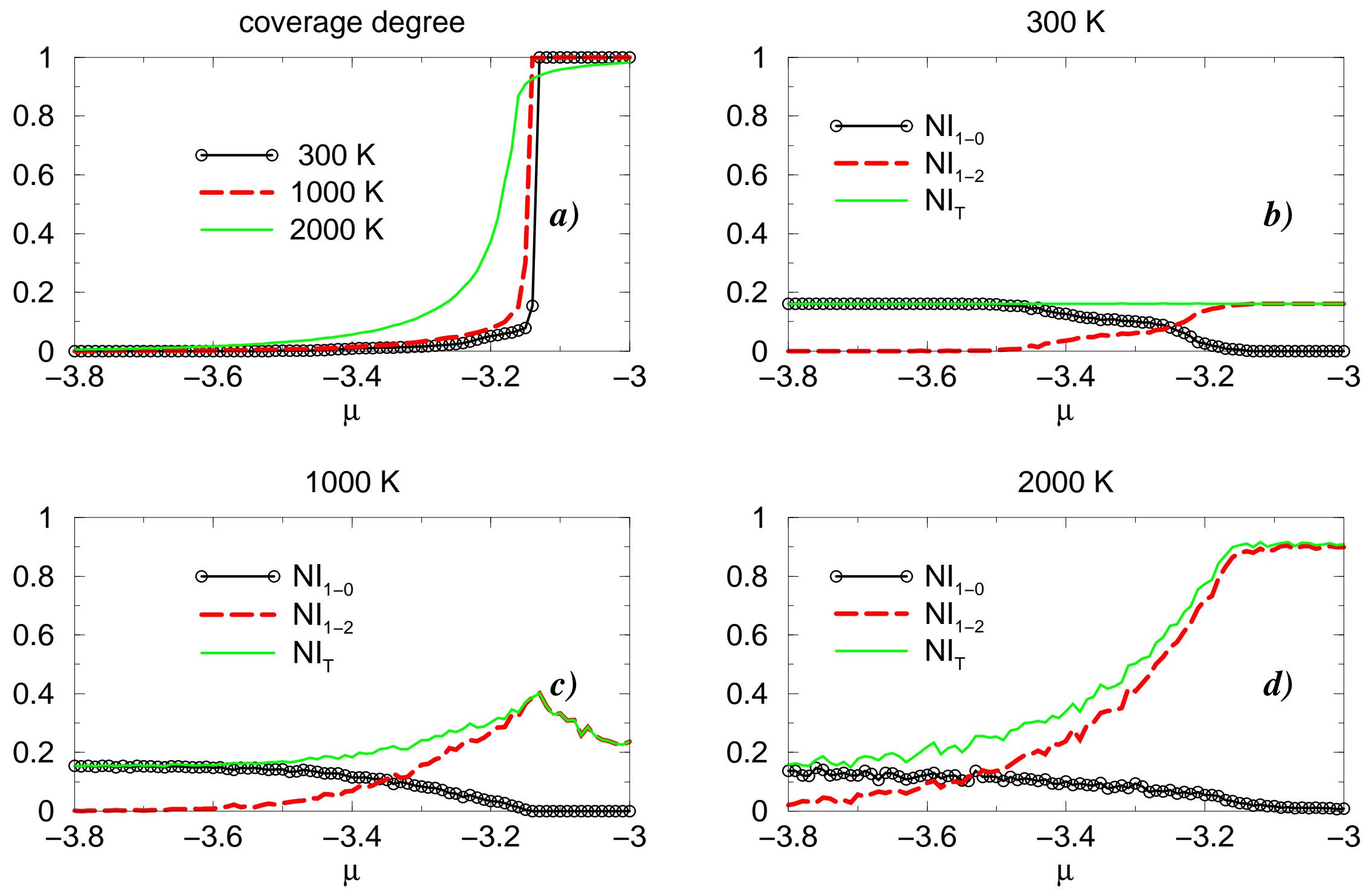

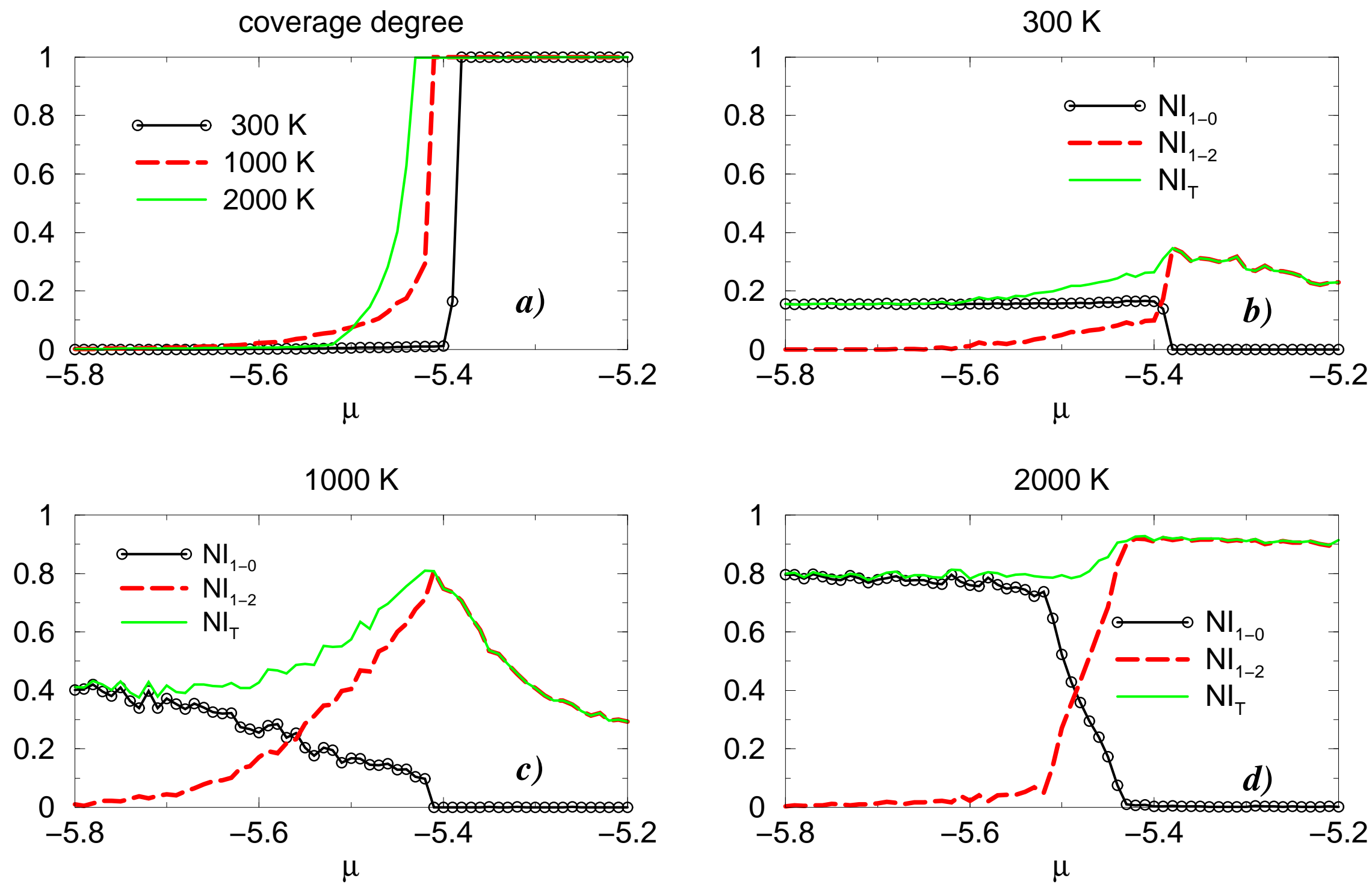